\title{
Segmentation and Free Space Detection Using Gabor Filters
}

\author{
Tadayoshi Shioyama ${ }^{1}$, Haiyuan $\mathrm{Wu}^{2}$, Masaya Takebe ${ }^{1}$, and Naoya Shimaoka ${ }^{1}$ \\ 1 Department of Mechanical and System Engineering \\ Kyoto Institute of Technology \\ Matsugasaki, Sakyo-ku, Kyoto 606-8585, Japan \\ shioyama@ipc.kit.ac.jp \\ 2 Department of Computer and Commucation Sciences \\ Wakayama University
}

\begin{abstract}
This paper proposes a new segmentation method and applies it for detecting free space, which is defined as a free road area without any obstacle. The problem is important for an intelligent transportation control or collision avoidance in vehicles, and also important for assisting the blind pedestrian. In this paper, the texture analysis is carried out using Gabor filters. Input images are segmented with not only the outputs of Gabor filters, but also both edge and color information. A free space is extracted from the segmented image. Then, the three-dimensional (3-D) information such as a free path at arbitrary direction from the observer is computed by stereo method. In order to evaluate the effectiveness of this proposed method, experimental results are shown for real images consisting of outdoor and indoor scenes.
\end{abstract}

\section{Introduction}

This paper proposes a new method for segmentation and applies it to free space detection. A free space is defined as a road area without any obstacle. A detection of free space is important for an intelligent transportation control [1] or collision avoidance in vehicles, and also important for assisting the blind pedestrian. For the purpose of free space detection, the first important task is to segment input images. In previous works on segmentation, methods using optical flow vectors [2]-[8] have been proposed to detect moving vehicles, and a method using Gabor filters [10] has been proposed to segment an image into an object and a background. These methods have not aimed at detecting a free space. A method based on a texture analysis using a decay autocorrelation function [9] has been proposed to detect a free space. However, this has not obtained the $3-\mathrm{D}$ information such as a free path from the observer to an obstacle, which is defined as the length without obstacle.

In this paper, we propose a new method for segmentation using Gabor filters [10][11] and using a new devised criterion. The goal of this research is to develop a method for detecting the free space. Hence the segmentation method is applied to detecting the free space from stereo images. The area in an image corresponding 
to free space is considered to be uniform in texture features. Texture analysis is carried out using Gabor filters operated on a grayscale image. The outputs of Gabor filters give information about local texture analysis. Input images are segmented with not only the outputs of Gabor filters, but also both edge and color information. Assuming that the lowest segment in an image corresponds to the road area where an observer stands, a free space is extracted from the segmented image. Then, the three-dimensional (3D) information such as a free path for arbitrary direction from the observer to an obstacle is computed by stereo measurement. To evaluate the proposed method, experimental results are shown for real images.

\section{Gabor Transformation}

Let $f(x, y)$ be the intensity at the coordinate $(x, y)$ in a gray scale image. Denoting by $\theta$ the counter clockwise angle from the positive $x$-axis, the Gabor transformation $z(x, y)$ of $f(x, y)$ in the direction of $\theta=0^{\circ}$ is given by

$$
z(x, y)=\int_{-\infty}^{\infty} f(x-t, y-s) \exp \left\{-\frac{1}{2}\left(\frac{t^{2}}{\sigma_{x}^{2}}+\frac{s^{2}}{\sigma_{y}^{2}}\right)\right\} \times \cos \left(2 \pi u_{0} t\right) d t d s
$$

where $u_{0}$ represents the radial frequency and $\sigma_{x}$ and $\sigma_{y}$ the space constants of the filter. The Fourier transformation of equation (1) is as follows:

$$
\begin{gathered}
Z(u, v)=F(u, v) G(u, v), \\
G(u, v)=A\left(\exp \left\{-\frac{1}{2}\left[\frac{\left(u-u_{0}\right)^{2}}{\sigma_{u}^{2}}+\frac{v^{2}}{\sigma_{v}^{2}}\right]\right\}+\exp \left\{-\frac{1}{2}\left[\frac{\left(u+u_{0}\right)^{2}}{\sigma_{u}^{2}}+\frac{v^{2}}{\sigma_{v}^{2}}\right]\right\}\right), \\
Z(u, v)=\int_{-\infty}^{\infty} \int_{-\infty}^{\infty} z(x, y) \times \exp \{-2 \pi i(u x+v y)\} d x d y \\
F(u, v)=\int_{-\infty}^{\infty} \int_{-\infty}^{\infty} f(x, y) \times \exp \{-2 \pi i(u x+v y)\} d x d y
\end{gathered}
$$

where $\mathrm{A} \equiv 2 \pi \sigma_{x} \sigma_{y}, \sigma_{u} \equiv 1 / 2 \pi \sigma_{x}, \sigma_{v} \equiv 1 / 2 \pi \sigma_{y}$ and $i \equiv \sqrt{-1}$. The Gabor filter is a band pass filter near the spatial frequency point $\left( \pm u_{0}, 0\right)$ as shown in equation (3). The Gabor transformation with arbitrary orientation $\theta$ is obtained from the filter by rotation of its $x-y$ coordinates. The output of the filter represents the energy at spatial frequency $u_{0}$ in an orientation $\theta$ in the input image.

\section{$3 \quad$ Feature Vectors}

Let $z\left(x, y ; u_{0}, \theta\right)$ be the output of a selected Gabor filter with parameters $u_{0}$ and $\theta$. To reduce the influence of the lighting condition, we normalize the output 
of Gabor filter about each direction. In the sequel, we use a transformation $P\left(x, y ; u_{0}, \theta\right)$ to which $z\left(x, y ; u_{0}\right.$, thet $)$ is subject:

$$
P\left(x, y ; u_{0}, \theta\right)=\frac{\left|z\left(x, y ; u_{0}, \theta\right)\right|}{\sum_{\theta \in \Theta}\left|z\left(x, y ; u_{0}, \theta\right)\right|} .
$$

The function $P\left(x, y ; u_{0}, \theta\right)$ takes the value in the range $[0,1]$ and represents the intensity at the frequency $u_{0}$ in the orientation $\theta$. For notational convenience, we denote by $P\left(u_{0}, \theta\right)$ the function $P\left(x, y ; u_{0}, \theta\right)$. Parameter $\theta$ is in $\Theta \equiv\left\{0^{\circ}, 45^{\circ}, 90^{\circ}, 135^{\circ}\right\}$

Since our aim is extracting the free road area, it does not require the minute information of the road surface. Therefore, we use the frequency component on the low area preferably. However, when Gabor filters are arranged on an extremely low area, the filters of 4 directions overlap each other, and then it is conceivable that the efficiency of texture analysis falls off. Under this opinion, the parameter $u_{0}$ is set as $u_{0}=1 / 16, \sigma_{x}=\sigma_{y}=3$, which is determined from both filter arrangement and experimental results of segmentation. Figure 1 shows an example of $P\left(u_{0}, \theta\right)$.

The feature vector $\vec{P}(x, y)$ at a coordinate $(x, y)$ in an image is constructed from $P\left(u_{0}, \theta\right)$ as follows:

$$
\vec{P}(x, y)=\left\{P\left(u_{0}, \theta\right), \theta \in \Theta, \text { and } S\right\}, \quad \text { where } S \equiv \sqrt{S_{x}^{2}+S_{y}^{2}},
$$

$S_{x}$ and $S_{y}$ are the outputs of $x$-directional Sobel operator, and $y$-directional Sobel operator, respectively. The edge information of outputs of Sobel operator is used for the purpose of discriminating two similar but different regions such as floor and wall with same color in indoor scene.

\section{Segmentation for Extracting the Free Space}

\subsection{Segmentation}

To improve the accuracy of segmentation, we do segment by using both the feature vector $\vec{P}(x, y)$ and a color vector $\vec{C}(x, y)$. The color vector $\vec{C}(x, y)$ is defined as the vector whose components are given by $(S \cos H, S \sin H)$ from the polar coordinates $(S, H)$ in the H-S plane in the HLS (Hue-Lightness-Saturation) color model.

In this paper, we merge similar regions neighboring to a considered region to carry out the segmentation. First, the initial regions are generated in the following steps:

(1) Assign a label to an unlabelled pixel found by scanning.

(2) Compute the distance $D^{2}$ between the last labeled pixel and its 4-neighboring pixel, and assign the same label to the pixel where the distance is less than a threshold $T_{1}(=0.01)$;

$$
D^{2} \equiv\|\vec{P}(a, b)-\vec{P}(c, d)\|^{2}+\eta\|\vec{C}(a, b)-\vec{C}(c, d)\|^{2},
$$




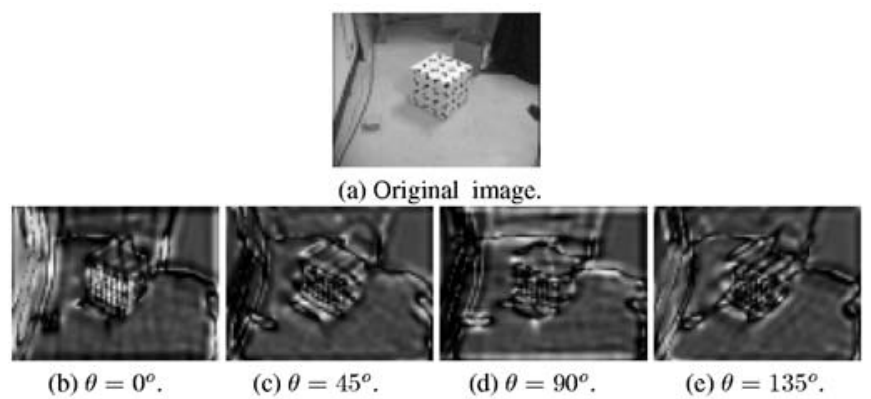

Fig. 1. An example of $P\left(u_{0}, \theta\right): u_{0}=1 / 16$.

where $(a, b)$ and $(c, d)$ are the coordinates of pixels in an original image, and $\eta$ denotes a weighting coefficient $(=0.2)$.

(3) Iterate the same process as the step (2) for the pixel, which is assigned the label in the step (2). When there is no pixel, which is to be assigned the same label, go to the step (4).

(4) If all pixels are already labeled, then stop. Otherwise, return to step (1).

Then, for each initial region, we compute the average of the feature vector, and also compute the center of gravity and the second central moments such as covariance from the coordinates of pixels belonging to the region. Utilizing this information, we efficiently merge regions by the following algorithm:

(1) For each region i, find a candidate region for merging, which satisfies the following condition:

$$
\begin{gathered}
\min _{j} E_{i j} \equiv U_{i j}^{2}\left\{R_{i j}+\kappa\left(M_{i j}^{2}+M_{j i}^{2}\right)\right\}, \\
U_{i j}^{2} \equiv\left\|\vec{P}_{i}-\vec{P}_{j}\right\|^{2}+\eta\left\|\vec{C}_{i}-\vec{C}_{j}\right\|^{2}<T_{2},
\end{gathered}
$$

where $\kappa(=1000)$ and $T_{2}(=0.2)$ are constants, and $\vec{P}_{i}, \vec{C}_{i}$ is the average of $\vec{P}, \vec{C}$ in region $i$, respectively,

$$
R_{i j} \equiv n_{i} n_{j} /\left(n_{i}+n_{j}\right),
$$

$n_{i}$ is the number of pixels in region $\mathrm{i}$,

$M_{i j}^{2} \equiv \frac{1}{\left(1-\rho_{i}^{2}\right) V_{x_{i}} V_{y_{i}}}\left\{V_{y_{i}}\left(x_{i}-x_{j}\right)^{2}-2 \rho_{i} \sqrt{V_{x_{i}} V_{y_{i}}}\left(x_{i}-x_{j}\right)\left(y_{i}-y_{j}\right)+V_{x_{i}}\left(y_{i}-y_{j}\right)^{2}\right\}$,

where, in region $i,\left(x_{i}, y_{i}\right)$ is the center of gravity, $V_{x_{i}}, V_{y_{i}}$ are variances in $x$-axis and $y$-axis, $\rho_{i}$ is correlation coefficient. $M_{i j}^{2}$ is Maharanobis distance from region $i$ to region $j$.

(2) Merge two regions, which are mutually candidates, each other. Compute the average of feature vector and the second central moments for the new region after merging.

(3) Iterate the procedure of steps (1) and (2) until there is no region which satisfies the condition (7). 
The criterion $E_{i j}$ includes the factor of similarity $U_{i j}^{2}$, the size factor $R_{i j}$ and the Maharanobis distance $M_{i j}^{2}$ which depends on the shape of the region. In this algorithm, the region with small size (i.e. small $R_{i j}$ ) is superiorly merged.

An example of segmentation by the above algorithm is shown in Fig. 2. Figures 2(b) and (c) show the segmentations at the initial stage and the final stage, respectively as the average intensity of each region.

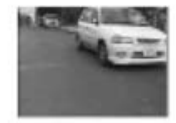

(a) Original image.

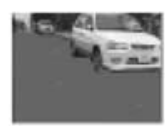

(b) At the initial stage.

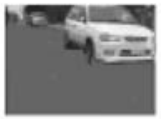

(c) At the final stage.

Fig. 2. Results of segmentation: $T_{1}=0.01, T_{2}=0.2, \eta=0.2, \kappa=1000$.

\subsection{Extraction of Free Space}

Based on the assumption that the lowest segment in an image corresponds to the road area where an observer stands, a free space can be extracted from the segmentation result. Some detected free spaces are illustrated in Figs. 3. The upper row is original images for real indoor and outdoor scenes. The figure on the left is in the case where the road is seen over a fence with net. The figure on the right is in the case where the person as the obstacle is standing in a dim corridor. The second row is binary images, and the detected free spaces are shown as white.

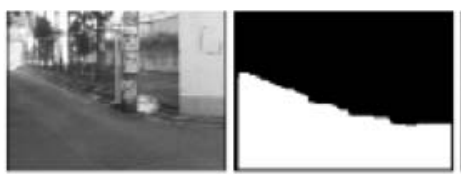

(1) an outdoor scene

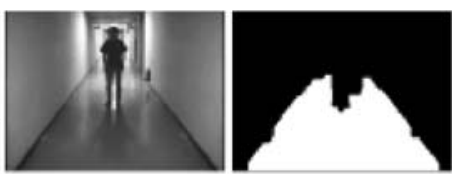

(2) an indoor scene

Fig. 3. Some detected free spaces.

\section{Measurement of Free Path}

In order to obtain 3D information about the free path and free direction, we find out corresponding points on the boundaries of free spaces in the stereo images. Here, we assume that the $3 \mathrm{D}$ plane of the road surface is known at the stereo camera calibration. 


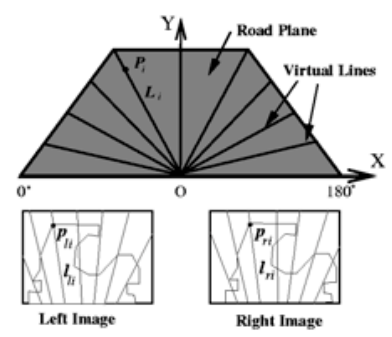

Fig. 4. Corresponding points on a free space boundary.

\subsection{Stereo Measuring}

To estimate the distance from the observer to the free space boundary from stereo images, it is necessary to solve the point correspondence. However, it is difficult to solve the point correspondence because there is no feature point on the free space boundary. Hence we find corresponding points on the boundary by the following method.

We set a $(X, Y, Z)$ coordinate system whose origin $O$ is on the road surface and it coincides with the point vertically projected on the road from the center of stereo cameras. Here, the $X-Y$ plane coincides with the road surface. The $X$-axis is set as parallel to the line connecting both the centers of stereo cameras.

On the $3 \mathrm{D}$ road surface, we consider the every $10^{\circ}$ virtual radial lines $L_{i}, i=1,2, \ldots, n$, passing through the origin $O$, and draw the lines $\ell_{\ell i}$ and $\ell_{r i}$ corresponding to $L_{i}$ in the left and right stereo images, respectively. We denote by $p_{\ell i}$ and $p_{r i}$ the intersections of lines $p_{\ell i}$ and $p_{r i}$ with the boundaries of free spaces in the stereo images, respectively. From the intersections $p_{\ell i}$ and $p_{r i}$, the $3 \mathrm{D}$ coordinates of the point $P_{i}$ corresponding to the two points is computed by stereo measurement. If the coordinate of the point $P_{i}$ (i.e. the height of the point from the road surface) is less than a threshold, then the point $P_{i}$ is considered to be on the line $L_{i}$ in the road surface. Here, the free path is defined as the horizontal distance from the observer to the boundary of the free space, and the direction is defined as an clockwise angle in degree from the negative $X$-axis on the road surface. So, the free path on the radial line $L_{i}$ is given by the distance $\overline{O P_{i}}$ from the origin $O$ to the point $P_{i}$.

However, this method is not able to do exact correspondence in the case that there is an obstacle out of touch with the road. To supplement this problem, we carry out the following method. When the height of the point $P_{i}$ is greater than a threshold, we shift the road surface at vertical direction hypothetically, and draw the hypothetical radial lines to do correspondence once again. The hypothetical road surface is shifted to the height of 2 meters every $1 \mathrm{~cm}$ until exact correspondence. 


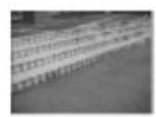

(a) Intensity images.
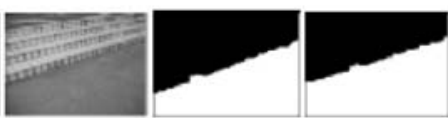

(b) Free spaces.

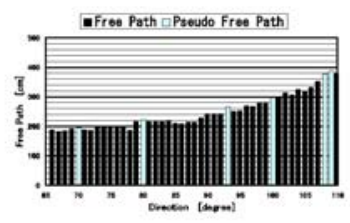

(c) Free path vs direction.

Fig. 5. The free space and free path.

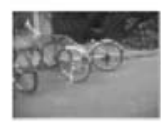

(a) Intensity images.
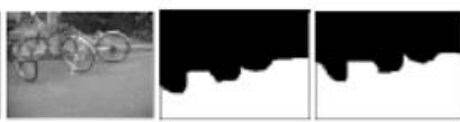

(b) Free spaces.

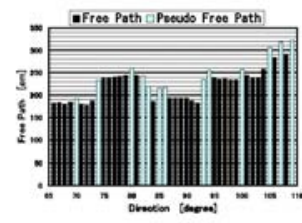

(c) Free path vs direction.

Fig. 6. The free space and free path.

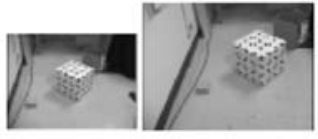

(a) Intensity images.

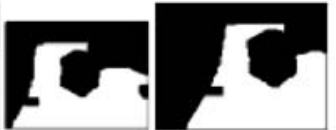

(b) Free spaces.

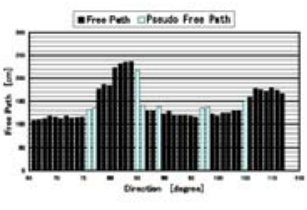

(c) Free path vs direction.

Fig. 7. The free space and free path.

\subsection{Monocular Measuring}

Free path corresponding to the point $p_{\ell i}$ and $p_{r i}$ may not be able to be calculated from the method above mentioned. In this case, one of the reasons for this failure in correspondence is that the boundary point $P_{i}$ is not visible from the left camera while it is visible from the right camera because of any obstacle and

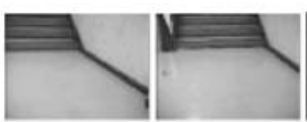

(a) Intensity images.

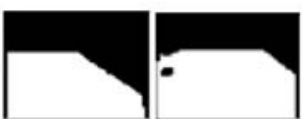

(b) Free spaces.

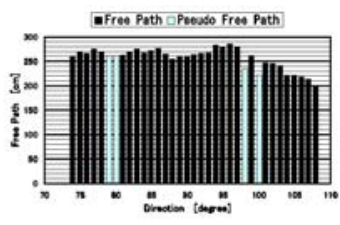

(c) Free path vs direction.

Fig. 8. The free space and free path. 


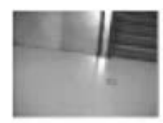

(a) Intensity images.

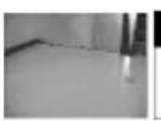

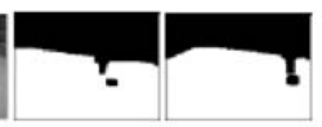

(b) Free spaces.

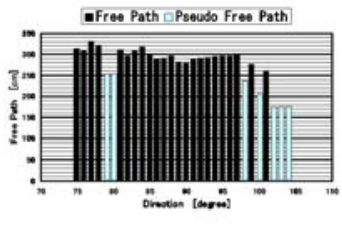

(c) Free path vs direction.

Fig. 9. The free space and free path.

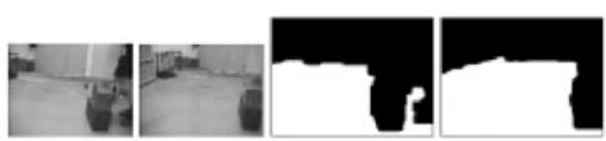

(a) Intensity images.

(b) Free spaces.

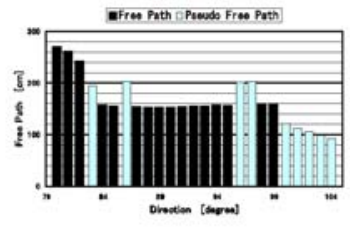

(c) Free path vs direction.

Fig. 10. The free space and free path.

vice versa. Another reason for such correspondence failure is that if the error in segmentation and free space boundary estimation is not small, then the error may result in the correspondence failure.

Hence for the convenience in the case of correspondence failure, we compute the $3 \mathrm{D}$ coordinate of the point on the road surface corresponding to the point $p_{\ell i}$ by the left camera calibration parameters and also compute that for $p_{r i}$ by the right camera parameters. Then we give the minimum value among the distances to these two points, as a pseudo free path for the purpose of giving the safer pseudo free path than free path.

\section{Experimental Results}

We tested our algorithm on some stereo images, which consist of indoor and outdoor scenes. Some results are shown in Figs. 5-10. In the figures, (a) shows the original images, (b) shows the detected free spaces and (c) shows the free path and pseudo free path against direction. The horizontal axis shows the free direction [degree], and the vertical axis shows free distance [cm]. Also, the black sticks express the free path and gray sticks express the pseudo free path. Figures 5 and 6 show the results for outdoor scenes of up steps and bicycles, respectively. Figures 7 through 10 show those for indoor scenes of room, up steps and down steps, respectively. From these results, it is found that the free space is effectively extracted. The RMS errors were $11[\mathrm{~cm}]$ in the case of indoor, and $47[\mathrm{~cm}]$ in the case of outdoor scenes, respective. 


\section{Conclusions}

We have proposed a new method for segmentation and applied it to detecting free spaces from input stereo images. The local texture analysis of a gray scale image has been carried out using Gabor filters. For the purpose of segmentation, the feature vectors have been constructed from not only the outputs of Gabor filters but also the color information and Soble filter of an original image. In order to effectively merge the pixels with similar features, the criterion has been devised so as to consider the factor of similarity, the size factor of the merged region and the shape factor of the region. A free space has been extracted from the segmented image assuming that the lowest segment in an image corresponds to the road area where an observer stands. The 3D information such as free paths has been computed by stereo measurement. From experimental results based on real scenes, it is found that the proposed method can effectively detect free spaces.

\section{References}

[1] Onoguchi, K., Takeda, N. and Watanabe, M., "Obstacle Location Estimation Using Planar Projection Stereopsis Method," IEICE Trans., Vol.J81-D-II, No.8 (1998) pp. 1895-1903.

[2] Adiv, G., "Determining Three-Demensional Motion and Structure from Optical Flow Generated by Several Moving Objects," IEEE Trans. on PAMI, Vol.7,No.4 (1985) pp. 384-401.

[3] Thompson, W.B., Mutch, K.M. and Berzins, V.A., "Dynamic Occlusion Analysis in Optical Flow Fields," IEEE Trans. on PAMI, Vol.7, No.4 (1985) pp. 374-383.

[4] Murray, D.W. and Buxton, B.F., "Scene Segmentation from Visual Motion Using Global Optimization," IEEE Trans. on PAMI, Vol.9, No.2 (1987) pp. 220-228.

[5] Thompson, W.B., Lechleider, P. and Stuck, E.R., "Detecting Moving Objects Using the Rigidity Constraint," IEEE Trans. on PAMI, Vol.15, No.2 (1993) pp. $162-166$.

[6] Ohta, N., "Structure from Motion with Confidence Measure and Its Application for Moving Object Detection," IEICE Trans., Vol. J76-D-II, No.8 (1993) pp. 15621571.

[7] Smith, S.M., "ASSET-2:Real-Time Motion Segmentation and Shape Tracking," Proc. of ICCV'95 (1995) pp. 237-244.

[8] Tian, T.W. and Shah, M., "Recovering 3D Motion of Multiple Objects Using Adaptive Hough Transform," Proc. of ICCV'95 (1995) pp. 284-289.

[9] Adorni, G. and Cagnoni, S., "A cellular automata-based tool as an aid for autonomous navigation," Proc. of 11th Scandinavian Conf. on Image Analysis, (1999) pp. 609-614.

[10] Jain, A.K., Ratha, N.K. and Lakshmanan, S., "Object Detection Using Gabor Filters," Pattern Recognition, Vol.30, No.2 (1997) pp. 295-309.

[11] Mitani, S., Wu, H. and Shioyama, T., "Car Detection with Gabor Filters," IEICE Trans., Vol.J83-D-II, No.12 (2000) pp. 2641-2651. 